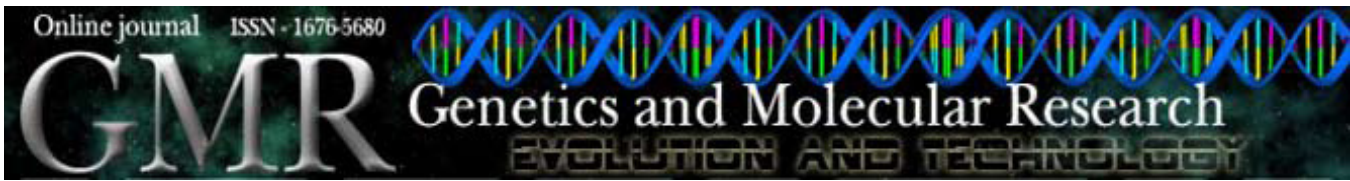

\title{
The role of disulfide bridges in the 3-D structures of the antimicrobial peptides gomesin and protegrin-1: a molecular dynamics study
}

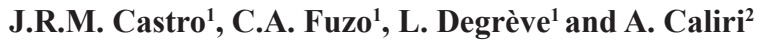 \\ ${ }^{1}$ Departamento de Química, Faculdade de Filosofia Ciências e Letras de \\ Ribeirão Preto, Universidade de São Paulo, Ribeirão Preto, SP, Brasil \\ ${ }^{2}$ Departamento de Física e Química, Faculdade de Ciências Farmacêuticas de \\ Ribeirão Preto, Universidade de São Paulo, Ribeirão Preto, SP, Brasil \\ Corresponding author: J.R.M. Castro \\ E-mail: jrcastro@usp.br
}

Genet. Mol. Res. 7 (4): 1070-1088 (2008)

Received August 6, 2008

Accepted August 27, 2008

Published October 14, 2008

\begin{abstract}
Some antimicrobial peptides have a broad spectrum of action against many different kinds of microorganisms. Gomesin and protegrin-1 are examples of such antimicrobial peptides, and they were studied by molecular dynamics in this research. Both have a $\beta$-hairpin conformation stabilized by two disulfide bridges and are active against Gram-positive and Gram-negative bacteria, as well as fungi. In this study, the role of the disulfide bridge in the maintenance of the tertiary peptide structure of protegrin- 1 and gomesin is analyzed by the structural characteristics of these peptides and two of their respective variants, gomy4 and proty4, in which the four cysteines are replaced by four tyrosine residues. The absence of disulfide bridges in gomy4 and proty4 is compensated by overall reinforcement of the original hydrogen bonds and extra attractive interactions between the aromatic rings of the tyrosine residues. The net effects on the variants with respect to the corresponding natural peptides are: i) maintenance of the original $\beta$-hairpin conformation, with great structural similarities between the mutant and the corresponding natural peptide; ii) combination of positive $\Phi$ and $\Psi$ Ra-
\end{abstract}


machandran angles within the hairpin head region with a qualitative change to a combination of positive $(\Phi)$ and negative $(\Psi)$ angles, and iii) significant increase in structural flexibility. Experimental facts about the antimicrobial activity of the gomesin and protegrin-1 variants have also been established here, in the hope that the detailed data provided in the present study may be useful for understanding the mechanism of action of these peptides.

Key words: Gomesin; Protegrin-1; Antimicrobial peptides; Cysteine-rich peptides; Molecular simulations

\section{INTRODUCTION}

The use of antimicrobial peptides by many organisms as part of their innate defense system against harmful invading microorganisms is widely recognized (Che et al., 2008). One peculiar characteristic of such peptides is their broad spectrum of action against different types of bacteria, as well as some fungi. The possible link between their versatility and the antimicrobial resistance phenomenon has led to many investigations of these systems.

Antimicrobial resistance is a serious problem faced by humanity nowadays; antibiotic-resistant microorganisms are a menace that leads to death all over the world. The impact of such a challenging problem has motivated numerous academic and pharmaceutical industry research groups to develop new drugs capable of dealing with the adaptation strategy (namely the selection of organisms adapted to some specific medium) that these organisms develop over time (Prates and Bloch Júnior, 2000; Fazio et al., 2006; Ramamoorthy et al., 2006; Che et al., 2008).

There are several reasons for the appearance of resistant bacterial strains. The human lifestyle itself (Daffre et al., 2001) is certainly one of them, mainly because of the excessive and frequently inappropriate use of antibiotics. However, the observation of nature itself may provide some insight into how to find a solution to this problem. Indeed, due to the exposure of living beings to an environment where contact with pathogens is unavoidable, nature has endowed organisms with distinctive tools for survival (Chan et al., 2006) as part of their innate immunity system via evolution and natural selection. In this context, the production of antimicrobial peptides is an ancient evolutionary defense weapon (Zasloff, 2002) that allows living organisms such as plants and vertebrate and invertebrate animals, and some microorganisms (fungi and bacteria) to survive.

What hinders the use of such broad-spectrum activity peptides against infectious disease is their potential toxicity and hemolytic effects. Heavy investments in research that could improve the selectivity of these peptides are necessary, so that they can be made active against pathogenic microorganisms only. Attempts to overcome the fast selection of microorganisms resulting from antimicrobial peptides are also mandatory.

Gomesin (PCA-CRRLCYKQRCVTYCRGR) is a peptide isolated from the blood cells of the mygalomorph spider Acanthoscurria gomesiana. Its N- and C-termini consist of pyroglutamic acid (PCA) and an amidated arginine (Silva Jr. et al., 2000; Mandard et al., 2002), respectively. As for protegrin-1 (RGGRLCYCRRRFCVCVGR) it is found in porcine leukocytes, and its C-terminus position is an amidated arginine (Fahrner et al., 
1996; Lam et al., 2006). Both protegrin-1 and gomesin are cationic peptides. The former contains six arginine residues, while the latter has five arginine and one lysine residues, all of them protonated at physiological $\mathrm{pH}$. Gomesin is active against bacteria, fungi and parasites. It is structurally very similar to protegrin-1, as well as other peptides such as androctonin, polyphemusins, and tachyplesin I, and it adopts a well-defined $\beta$-hairpin-like structure (Fahrner et al., 1996; Fazio et al., 2006; Lam et al., 2006). Protegrin-1 is also an antimicrobial peptide, active against several Gram-positive and Gram-negative bacteria, fungi, and HIV-1. The molecular masses of gomesin and protegrin-1 are 2270 and 2154 $\mathrm{Da}$, respectively. Both have eighteen residues, four of which are cysteines constituting two disulfide bridges: Cys2-Cys15 and Cys6-Cys11 in gomesin; Cys6-Cys15 and Cys8Cys13 in protegrin-1. Using 2-D nuclear magnetic resonance spectroscopy, Mandard et al. (2002) proposed the gomesin tertiary structure, which is identified in the Protein Data Bank (PDB) by the code 1KFP (www.rcsb.org/pdb). Using the same technique, Fahrner et al. (1996) determined the tertiary structure of protegrin-1, identifying it by the PDB code 1 PG1.

In the present study, we proposed the use of two variants, namely proty 4 (protegrin- $1 \mathrm{mu}$ -

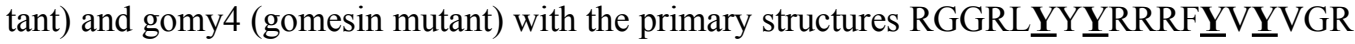
and PCA- $\underline{Y} R R L \underline{Y} Y K Q R \underline{Y} V T Y \underline{Y} R G R$, respectively, where the four cysteines are replaced by four tyrosines, in order to analyze the role of the disulfide bridges in the structural stability of these two peptides. Our proposition is based on the study carried out with tachyplesin-I mutants, in which the four existing cysteines of the natural peptide were replaced by tyrosine residues (Rao, 1999; Ramamoorthy et al., 2006). In the study with tachyplesin-I, the authors found a great similarity between the native and the mutant conformations. They also reported that the deletion of all cysteines abolished the hemolytic effect, but the antimicrobial activity remained unchanged.

The parameters and force field used in our studies describe the systems under consideration quite well; in fact, our structural and hydrogen bond (HB) data for gomesin and protegrin-1 are in very good agreement with experimental data. Details on conformational characteristics, HBs, and their evolutionary behavior are provided for both the natural peptides and their mutants.

The structural flexibility of the natural peptides - despite the presence of two disulfide bridges and several cross-strand HBs - may be the most important factor related to the broadspectrum antimicrobial action of these peptides. Therefore, since our results indicate that the proposed variants are structurally very similar to the corresponding natural peptides, and also predict a significant increase in flexibility for both variants, the detailed data provided in this study can aid in better understanding the role of the disulfide bridges in the secondary structures of these peptides.

\section{MATERIAL AND METHODS}

From the PDB structures and using the DeepView/Swiss-PdbViewer 3.7 software (Guex and Peitsch, 1997), it was possible to obtain the initial configurations of the two mutants. The construction of the systems and the molecular dynamics (MD) simulations were carried out using the GROMACS 3.3.1 (Van Der Spoel et al., 2005) package and GROMOS96 (Van Gunsteren et al., 1996) force field. The four systems were constructed 
placing the center of mass $(\mathrm{CM})$ of the peptides in the center of a cubic simulation box with edges equal to the peptide's largest interatomic distance plus $4 \mathrm{~nm}$. The box was filled with water molecules represented by the SPC model (Berendsen et al., 1987). Chloride ions were inserted into electrostatically favorable positions, in order to counterbalance the peptide charges, thus keeping the local electro-neutrality of the systems. The peptide covalent bonds were constrained by the LINCS algorithm (Hess et al., 1997), while the SETTLE algorithm (Miyamoto and Kollman, 1992) was used to maintain the water SPC molecules structurally stable. The temperature $(300 \mathrm{~K})$ and the pressure $(1 \mathrm{~atm})$ of the systems $(\mathrm{NpT}$ ensemble) were regulated by the Berendsen's algorithms (Berendsen et al., 1984), using correlation times of $0.1 \mathrm{ps}$. A cut-off of $1.0 \mathrm{~nm}$ on the non-bonded interactions was used. The long range electrostatic interactions were calculated using the particle mesh Ewald summation method (Darden et al., 1993). In order to eliminate bad contacts, the system energy was first minimized by the steepest descent algorithm. The simulation of each system was then carried out for $0.5 \mathrm{~ns}$, under peptide atomic coordinate restrictions with an integration time step of $0.5 \mathrm{fs}$. Next, the simulation of each system was carried out for an extra $0.5 \mathrm{~ns}$ still under peptide atomic coordinate restrictions, but using an integration time step of $1.0 \mathrm{fs}$. These two cycles of $0.5 \mathrm{~ns}$ each were performed in order to equilibrate the restricted system at a constant temperature of $300 \mathrm{~K}$. The MD simulations were then carried out without restrictions on peptide motion for 19 and $37 \mathrm{~ns}$ in the cases of gomesin and protegrin-1, respectively. Longer runs were used for the variants gomy4 (70 ns) and proty 4 (75 ns) in order to check the maintenance of the initially guessed $\beta$-hairpin conformation. In the productive stage, the integration time steps were fixed at $2 \mathrm{fs}$ for all cases.

The intramolecular HBs were identified from the atomic trajectories. In our calculations, an HB was considered to exist when the donor-acceptor distance was smaller than $0.35 \mathrm{~nm}$, and the angle between the hydrogen-donor and donor-acceptor vectors was smaller than 30 degrees. Only the occurrence of such conditions greater than $20 \%$ of the total simulation time was considered. The radius of gyration $(\mathrm{Rg})$ and the root mean square deviations (RMSD) were systematically used to analyze the structural, evolutionary behavior of the systems; the RMSD was obtained by least-square fitting of the peptide structures to the reference, which was the initial structure of each peptide in all systems. The Rg provides global information about structural packaging, while RMSD is a measure of the similarity between two structures. In these studies, the GROMACS (Van Der Spoel et al., 2004) analyzing tools package was employed.

For trajectory analysis, time intervals of 10-19 and 20-70 ns were used for gomesin and gomy4, respectively, while for protegrin-1 and proty4 they were 10-37 and 20-70 ns, respectively.

\section{RESULTS}

The structural characteristics of gomesin and gomy4, as well as those of protegrin-1 and proty4, were first analyzed by comparing their respective RMSDs. Figure 1 shows the alpha carbon $\left(\mathrm{C}_{\alpha}\right)$ RMSD evolution for all four cases. The time-averaged RMSD for gomesin and gomy 4 are $0.23 \pm 0.04$ and $0.26 \pm 0.04 \mathrm{~nm}$, respectively; while for protegrin-1 and proty 4 , average RMSD values of $0.25 \pm 0.06$ and $0.25 \pm 0.07 \mathrm{~nm}$ are obtained, respectively. Since the same backbone coordinates are used as reference for each corresponding peptide-variant pair, 
the comparatively small RMSD magnitude throughout the simulation suggests that a structural similarity is perennially maintained between the peptide and its variant.

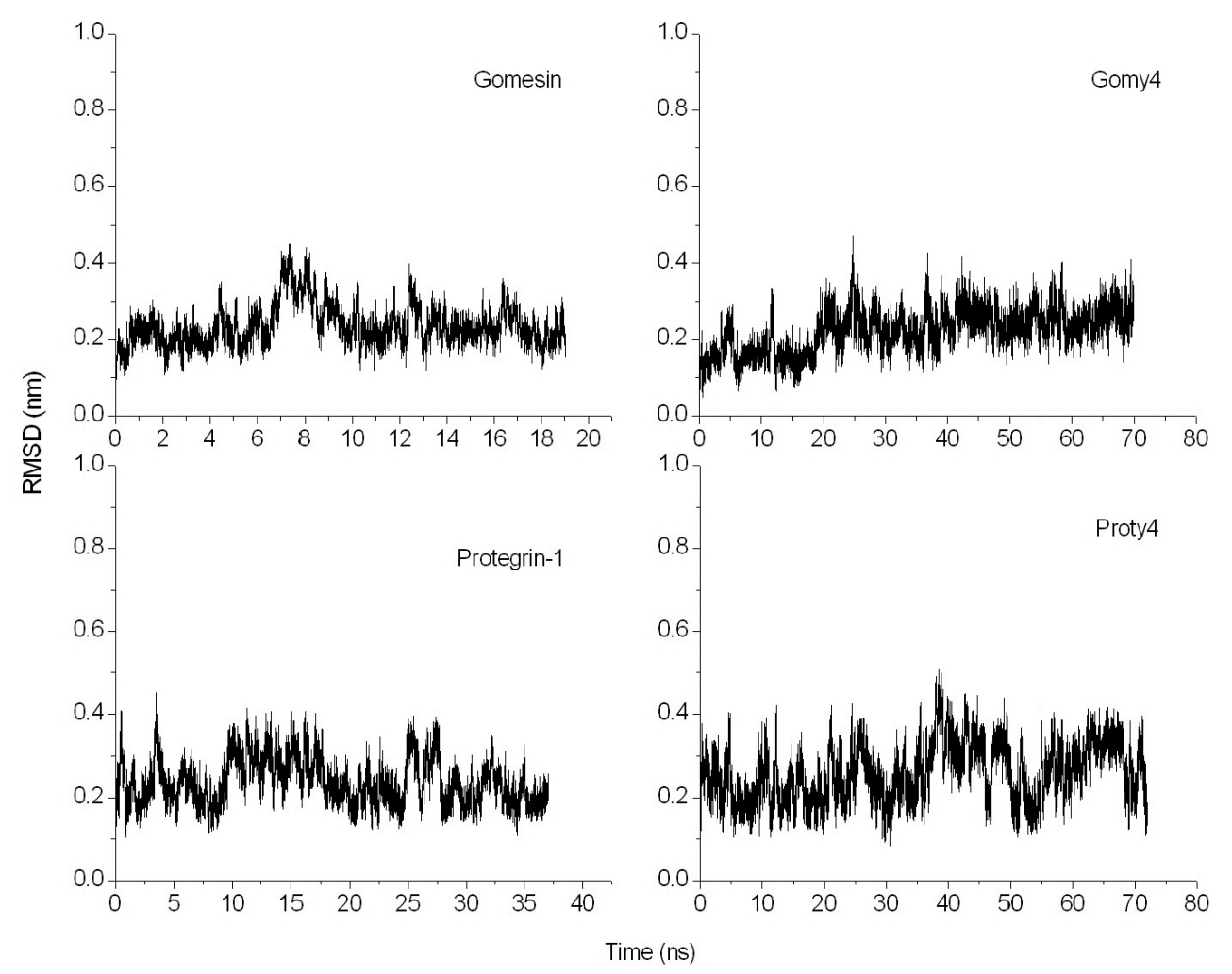

Figure 1. Alpha carbon-root mean square deviation $\left(\mathrm{C}_{\alpha}-\mathrm{RMSD}\right)$ evolution for gomesin, protegrin-1, gomy4 and proty4. For each corresponding peptide-mutant pair, the reference structure $\left(\mathrm{C}_{\alpha}\right)$ is the same.

The average $\mathrm{Rg}$ values for gomesin and gomy4 are $0.88 \pm 0.04$ and $0.87 \pm 0.04$ $\mathrm{nm}$, respectively. As for protegrin-1 and proty $4 \mathrm{Rg}$ values are equal to $0.89 \pm 0.04$ and $0.88 \pm 0.04 \mathrm{~nm}$, respectively. Such good combination of numerical congruence for the $\mathrm{Rg}$ and RMSD values is the first indication of the structural similarity between these corresponding peptide-variant pairs.

\section{The hydrogen bonds}

Fine details of the structural similarities are obtained from the HB analysis. First, the simulation results concerning the coupled peptide-variant pairs confirm that a peculiar pattern of cross-strand HBs significantly contributes to the stabilization of the $\beta$-hairpin-like structure adopted by gomesin (Mandard et al., 2002) and protegrin-1 (Fahrner et al., 1996) in aqueous solution. Tables 1 and 2 summarize the results for the cross-strand HBs found in our MD simulation for both natural peptides and their respective variants. 
Table 1. Protegrin-1 and proty4. Hydrogen bonds determined by molecular dynamics simulations.

\begin{tabular}{lcc}
\hline Hydrogen bonds & \multicolumn{2}{c}{ Occurrence (\%) } \\
\cline { 2 - 3 } & Protegrin-1 & Proty4 \\
\hline NH(Leu5) - CO(Val16) & 64.6 & 84.0 \\
NH(Val16) - CO(Leu5) & 80.1 & 76.5 \\
NH(Tyr7) - CO(Val14) & 80.2 & 89.8 \\
NH(Val14) - CO(Tyr7) & 76.8 & 90.7 \\
NH(Arg9) - CO(Phe12) & 67.4 & 83.7 \\
NH(Phe12) - CO(Arg10)* & 56.8 & - \\
NH(Arg18) - CO(Gly3)* & 34.6 & 58.3 \\
\hline
\end{tabular}

*These hydrogen bonds were not identified experimentally. For trajectory analysis, time intervals of 10-37 ns were used for protegrin-1, and 20-70 ns for proty4.

Table 2. Gomesin and gomy4. Hydrogen bonds determined by molecular dynamics simulations.

\begin{tabular}{lcc}
\hline Hydrogen bonds & & Occurrence (\%) \\
\cline { 2 - 3 } & Gomesin & Gomy4 \\
\hline NH(Tyr7) - CO(Arg10) & - & 74.9 \\
NH(Arg10) - CO(Tyr7) & - & 59.5 \\
NH(Arg16) - CO(PCA1) & 69.3 & - \\
NH(Arg3) - CO(Tyr14) & 76.0 & 89.1 \\
NH(Tyr14) - CO(Arg3) & 76.1 & 89.4 \\
NH(Leu5) - CO(Val12) & 81.0 & 90.6 \\
NH(Val12) - CO(Leu5) & 66.4 & 79.4 \\
\hline
\end{tabular}

In general, our results are in good agreement with the HB data and structural characteristics experimentally proposed for gomesin and protegrin-1 peptides (Fahrner et al., 1996; Mandard et al., 2002). The slight difference lies in the two extra HBs that appear in our simulations, namely the HBs formed by Phe12-Arg10 and Gly3-Arg18, which had not been experimentally observed for protegrin-1.

For the variants proty4 and gomy4, our MD simulations are also in agreement with the HBs proposed experimentally for the natural peptides (Fahrner et al., 1996; Mandard et al., 2002), with a significant increase in their occurrence when compared with the HBs of the respective natural peptides. The HB between Arg10 and Tyr7, as identified in ten of the twenty experimentally proposed structures (Mandard et al., 2002), was not detected during our MD simulations of gomesin, but it did appear in the simulation of gomy4 with an occurrence of $59.5 \%$.

In proty4, the occurrence of HB between Val16 and Leu5 is a little less than in protegrin-1, and the HB between Phe12 and Arg 10 is missing. On the other hand, all HBs detected by our MD simulation of gomesin are also found for gomy4, but an extra HB between Tyr7 and Arg10 only occurs in gomy4, Table 2. It is noteworthy that this HB between Tyr7 and Arg10 was experimentally detected in only ten of the twenty structures proposed for gomesin (Mandard et al., 2002).

These results draw attention to the significant role of cross-strand HBs in the maintenance of the structural stability of the mutants despite the absence of disulfide bridges in these mutant peptides. Indeed, it has been experimentally observed that the location of the disulfide bridges within the $\beta$-hairpin structure is a determinant factor for the $\beta$-hairpin stability only when disulfide 
bonds link originally non-hydrogen-bonded residues facing each other (Santiveri et al., 2008). Just in this case, do the disulfide bridges effectively contribute to the stabilization of the $\beta$-hairpin structure. However, it must be pointed out that these experimental data were not obtained with mutants where the cysteine residues were substituted by tyrosine residues. Accordingly, in the present case, we observe that the removal of the disulfide bridges and the introduction of tyrosine residues not only essentially preserve all original $\mathrm{HBs}$, but promote a generalized increase in $\mathrm{HB}$ occurrence even when missing HBs are considered, as in the case of proty 4.

\section{The Ramachandran angles}

We also calculated the Ramachandran angles for the two peptides and their variants. Experimental data for gomesin (Mandard et al., 2002) assert that both dihedral angles $\Phi$ and $\Psi$ for Lys8 are positive, while for its neighbor, Gln9, both $\Phi$ and $\Psi$ are negative. Our MD simulations found this motif in gomesin, $(\Phi, \Psi)_{\mathrm{Lys} 8}=\left(+50^{\circ},+75^{\circ}\right)$, and in protegrin- $1,(\Phi, \Psi)_{\operatorname{Arg} 10}=$ $\left(+55^{\circ},+75^{\circ}\right)$. Interestingly, these two residues occupy the position where the chain starts to fold to form the typical hairpin $\beta$-strand. Moreover, in these peptides there are some residues with two preferential $\Phi$ and $\Psi$ angles, as shown by the distribution of the Ramachandran angles in Figures 2 and 3. This supports the premise that such peptides exhibit more than one meta-stable conformation in aqueous solution, which may be correlated with their broad-spectrum of antibiotic action. Actually, these alternative conformations may reflect certain structural flexibility that is responsible for increasing the interaction efficiency between the peptides and different kinds of cell membrane.

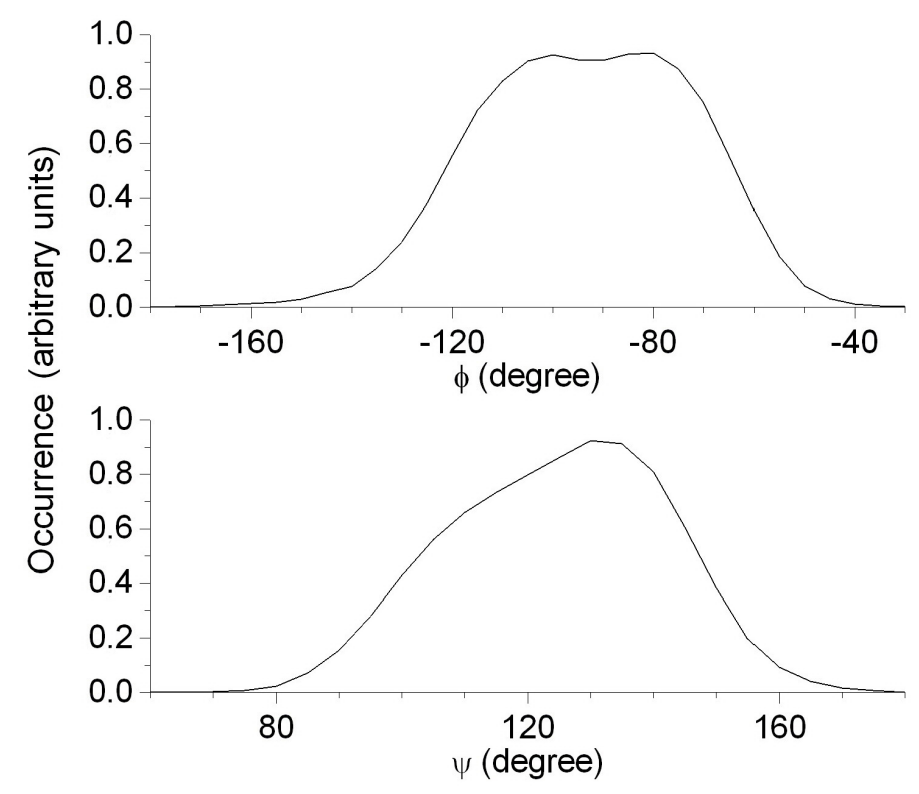

Figure 2. Protegrin-1. Distribution of the Ramachandran angles for Phe12, showing that the $\Phi$ angle has two near peaks at about $-100^{\circ}$ and $-80^{\circ}$, and a broad distribution of significant values for $\Psi$ angles in the interval between $90^{\circ}$ and $160^{\circ}$, with an asymmetric peak around $\Psi=135^{\circ}$. 

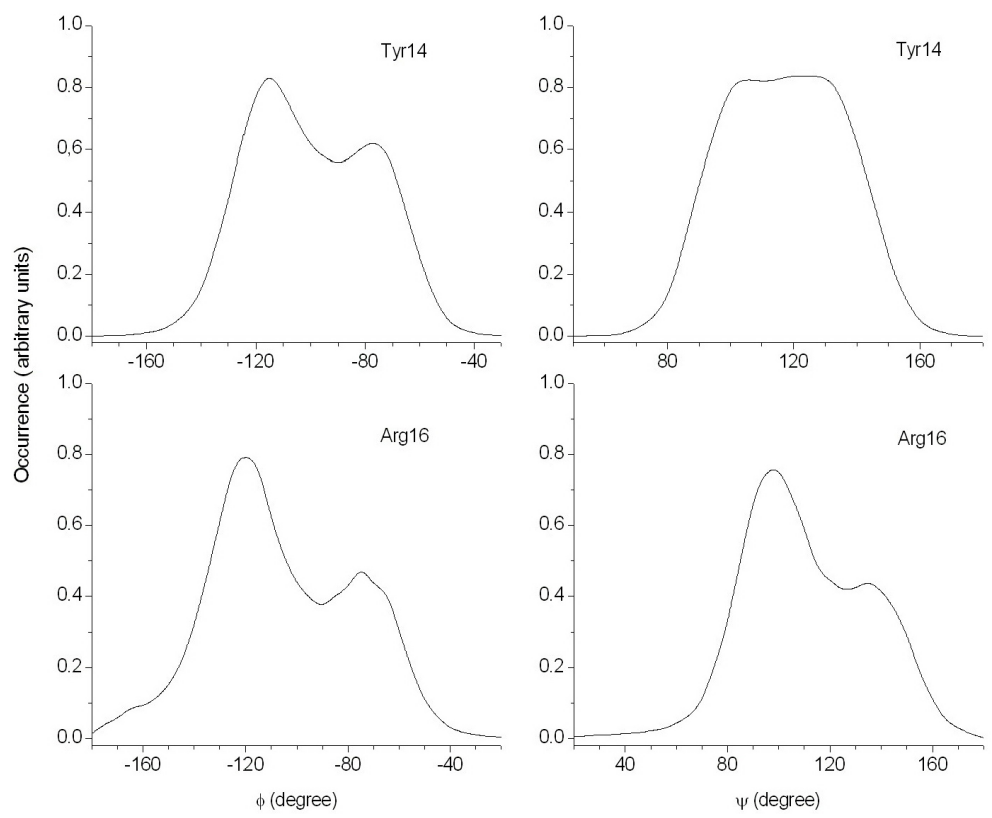

Figure 3. Gomesin. Distribution of the Ramachandran angles for two residues. For Tyr14, the distribution of $\Phi$ angles has two peaks at about $-115^{\circ}$ and $-75^{\circ}$, and two close peaks of $\Psi$ angle distribution at about $100^{\circ}$ and $130^{\circ}$. For $\operatorname{Arg} 16$, the peaks are about $\Phi=-120^{\circ}$ and $-75^{\circ}$, and $\Psi=100^{\circ}$ and $135^{\circ}$.

In the cases of both proty4 and gomy4, the original pairs of positive Ramachandran angles $\Phi$ and $\Psi$, as found in their corresponding natural peptides, undergo a qualitatively change: $\Phi$ is still a positive angle, but $\Psi$ changes into negative values: for $\operatorname{Arg} 10$ in proty4, the change is $(\Phi, \Psi)$ $=\left(+55^{\circ},+75^{\circ}\right) \rightarrow\left(+60^{\circ},-85^{\circ}\right)$; for Lys8 in gomy4, the change is $(\Phi, \Psi)=\left(+50^{\circ},+75^{\circ}\right) \rightarrow\left(+60^{\circ}\right.$, $\left.-80^{\circ}\right)$. These subtle differences deserve experimental investigation. Indeed, Fazio et al. (2006) have already studied several gomesin variants with respect to structure-activity relationship and the importance of disulfide bridges for conformation and bioactivity; however, the four cysteines were not replaced by tyrosine residues in any of the fourteen mutants studied by them.

Tables 3 and 4 show the peak maximum values or the shoulders in the Ramachandran angle histograms for gomesin/gomy4 and protegrin-1/proty4, respectively. It is interesting to note the reduction in the number of peaks in the mutant histograms. The rows in gray color indicate the residues that form HB. The absence of the disulfide bridges in the variants cause an increase in HB occurrence, as shown in Tables 1 and 2, and a considerable decrease in peak numbers in the Ramachandran angle histograms. This is one more proof that the disulfide bridges within the $\beta$-hairpin structure are a determinant factor for the $\beta$-hairpin stability only when disulfide bonds link originally non-hydrogen-bonded residues. The multimodal distributions of the Ramachandran angles are a consequence of the inadequacy of the disulfide bridge to reduce the tensions in its neighboring region (Santiveri et al., 2008).

Moreover, gomy 4 and proty 4 exhibit $\Phi$ and $\Psi$ values very close to the corresponding gomesin and protegrin-1 values, respectively. This is a strong evidence of the similarity between the variant and natural peptide structures. 
Table 3. Gomesin and gomy4 peaks in the Ramachandran angle histogram.

\begin{tabular}{|c|c|c|c|c|}
\hline \multirow[t]{2}{*}{ Residue } & \multicolumn{2}{|c|}{ Gomesin } & \multicolumn{2}{|c|}{ Gomy4 } \\
\hline & $\Phi$ (degree) & $\Psi$ (degree) & $\Phi$ (degree) & $\Psi$ (degree) \\
\hline Cys2 / (Tyr2)** & -80 & 95 & -70 & 95 \\
\hline Arg3 & $-120 ;-80^{*}$ & 145 & -115 & 145 \\
\hline $\operatorname{Arg} 4$ & -70 & 95 & -70 & 95 \\
\hline Leu5 & $-120 ;-80^{*}$ & 140 & -120 & 145 \\
\hline Cys6 / (Tyr6)** & -80 & 95 & -75 & 115 \\
\hline Tyr7 & $-120 ;-80^{*}$ & 100 & -120 & 105 \\
\hline Lys8 & 50 & 75 & 60 & -80 \\
\hline $\mathrm{G} \ln 9$ & 70 & -35 & -120 & -30 \\
\hline Arg10 & $-70 ;-120^{*}$ & 100 & $-125 ;-80^{*}$ & 145 \\
\hline Cys11 / (Tyr11)** & -85 & 90 & -80 & 95 \\
\hline Val12 & $-120 ;-80^{*}$ & 145 & -120 & 145 \\
\hline Thr13 & -70 & 95 & -70 & 100 \\
\hline Tyr14 & $-115 ;-75$ & $100 ; 130$ & -120 & 140 \\
\hline Cys15 / (Tyr15)** & -80 & 95 & -75 & 95 \\
\hline $\operatorname{Arg} 16$ & $-120 ;-75$ & $100 ; 135$ & -125 & 135 \\
\hline Gly17 & $75 ; 110$ & $-80 ;-150$ & $120 ;-60$ & $-80 ;-150 ; 80 ; 150$ \\
\hline
\end{tabular}

*It appears as a shoulder in the histograms. ${ }^{* *}$ Corresponding residue in the mutant. The gray color indicates the residues, which makes hydrogen bonds.

\begin{tabular}{|c|c|c|c|c|}
\hline \multirow[t]{2}{*}{ Residue } & \multicolumn{2}{|c|}{ Protegrin-1 } & \multicolumn{2}{|c|}{ Proty4 } \\
\hline & $\Phi$ (degree) & $\Psi$ (degree) & $\Phi$ (degree) & $\Psi$ (degree) \\
\hline Gly2 & $-115 ;-70 ; 65 ; 120$ & $-150 ;-90 ; 150 ; 90$ & $-115 ;-70 ; 65 ; 120$ & $-150 ;-90 ; 150 ; 90$ \\
\hline Gly3 & $-110 ;-65 ; 65 ; 115$ & $-145 ;-95 ; 145 ; 95$ & $-110 ;-65 ; 65 ; 115$ & $-145 ;-95 ; 145 ; 95$ \\
\hline $\operatorname{Arg} 4$ & $-70 ;-120 *$ & $95 ; 140^{*}$ & -70 & 100 \\
\hline Leu5 & $-110 ;-80^{*}$ & 135 & -120 & 150 \\
\hline Cys6 / (Tyr6)** & -75 & 100 & -75 & 100 \\
\hline Tyr7 & -110 & 130 & -120 & 145 \\
\hline Cys8 / (Tyr8)** & -70 & 95 & -80 & 100 \\
\hline $\operatorname{Arg} 9$ & $-115 ;-80^{*}$ & 100 & -120 & 100 \\
\hline Arg10 & 55 & 75 & 60 & -85 \\
\hline Arg11 & 70 & -35 & 65 & $20 ;-30$ \\
\hline Phe12 & $-80 ;-100$ & $130 ; 110^{*}$ & $-125 ;-75$ & 145 \\
\hline Cys $13 /(\operatorname{Tyr} 13)^{* *}$ & 75 & 100 & -90 & $105 ; 130^{*}$ \\
\hline Val14 & -105 & 125 & -120 & 145 \\
\hline Cys $15 /(\operatorname{Tyr} 15)^{* *}$ & -75 & 95 & -80 & 90 \\
\hline Val16 & -120 & 145 & -120 & 145 \\
\hline Gly 17 & $-65 ; 65 ; 125$ & $-150 ;-105 ; 95$ & $-120 ;-70 ; 55$ & $-40 ; 105 * ; 145$ \\
\hline
\end{tabular}

*It appears as a shoulder in the histograms. **Corresponding residue in the mutant. The gray color indicates the residues, which makes hydrogen bronds.

\section{Interatomic distances}

Our MD studies show that gomesin and its variant gomy4 are structurally very alike. It seems that the lack of disulfide bridges is fully compensated in some way. First, the HBs are strengthened, with an overall greater occurrence (Table 2). Second, the original C2-C15 and C6C11 bridges are substituted by efficient side-chain packing involving PCA1-Tyr15-Tyr2, and 
Tyr7-Tyr14. Specifically, Tyr15 interacts simultaneously with PCA1 and Tyr2, as seen in Figure 4, maintaining an average $\mathrm{CM}$ distance of about $0.5 \mathrm{~nm}$ from these two residues. Tyr7 also interacts attractively with the Tyr14 residue, as seen in Figure 5, with an average distance between the $\mathrm{CM}$ of the two aromatic rings of about $0.6 \mathrm{~nm}$ (Table 5). The resulting net effect of these strategic interactions is the energetic stabilization of the structure in an antiparallel $\beta$-sheet conformation.

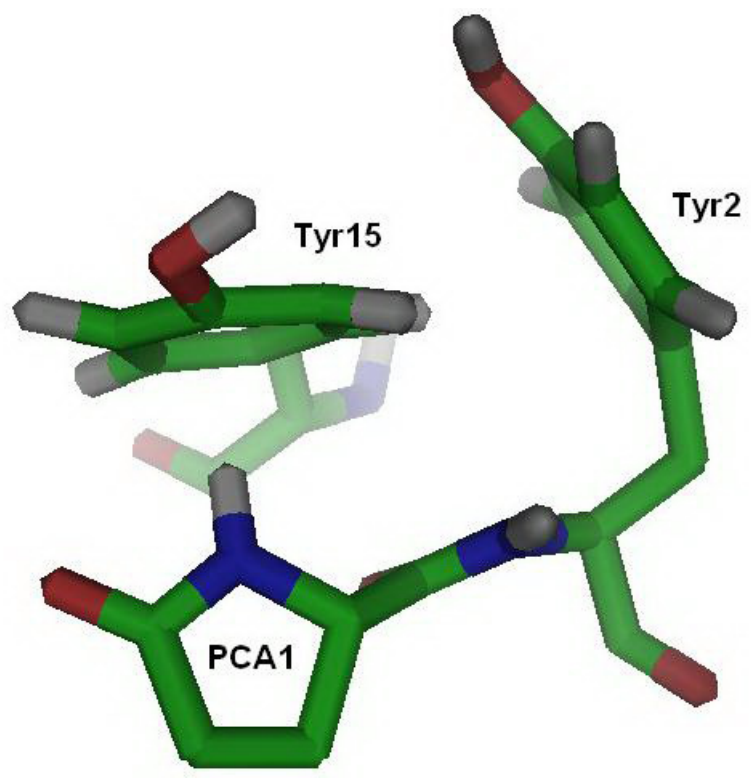

Figure 4. The PCA1, Tyr2 and Tyr15 region in gomy4. Interplanar angles between Tyr15-PCA1 and Tyr15-Tyr2: average of $87^{\circ}$ and $94^{\circ}$, respectively.

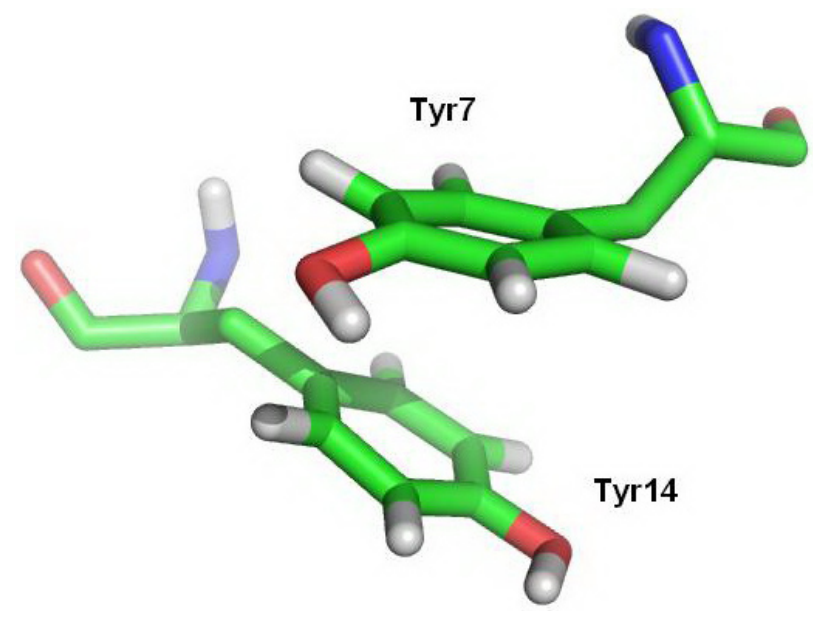

Figure 5. The Tyr7 and Tyr14 region of gomy4. Interplanar angle between Tyr7 and Tyr14: average of $21^{\circ}$. 


$\begin{aligned} & \text { Table 5. Gomy4. Average center of mass distances, interplanar angles, and interaction energies for pairs Tyr-Tyr } \\
& \text { and PCA-Tyr1. }\end{aligned}$
\begin{tabular}{lccc}
\hline Residues & Average center of mass distance (nm) & $\begin{array}{c}\text { Angles } \\
(\text { degree })\end{array}$ & $\begin{array}{c}\text { Average energy } \\
\left(\mathrm{kJ} \mathrm{mol}^{-1}\right)\end{array}$ \\
\hline PCA-Tyr15 & $0.5 \pm 0.3$ & $87 \pm 26$ & $-23 \pm 7$ \\
Tyr2-Tyr15 & $0.5 \pm 0.1$ & $94 \pm 32$ & $-15 \pm 3$ \\
Tyr7-Tyr14 & $0.6 \pm 0.1$ & $21 \pm 10$ & $-13 \pm 7$ \\
\hline
\end{tabular}

Now, for proty4, the pairs of residues (Tyr6, Tyr8) and (Tyr13, Tyr15) are separated by just one residue along the chain. There are significant attractive interactions not only in Tyr6-Tyr15 and Tyr8-Tyr13, but also between Tyr6 and Tyr13, since these residues are close (Figure 6).

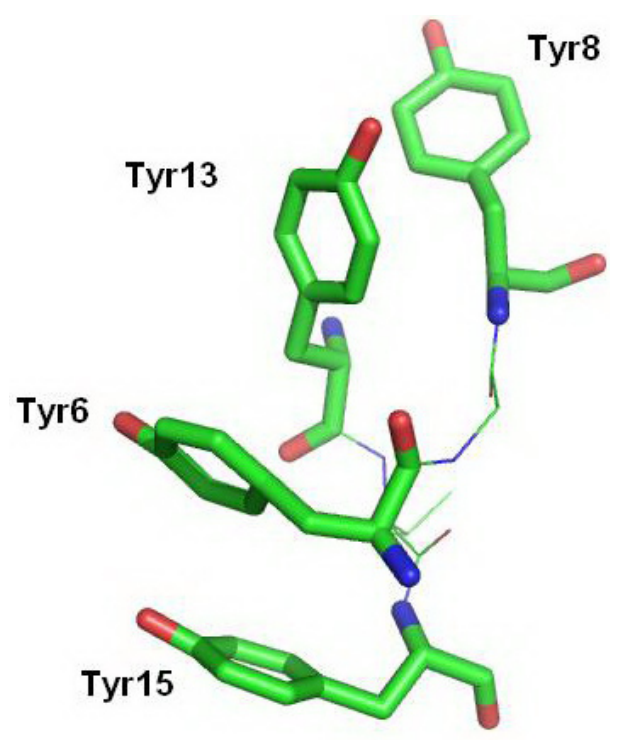

Figure 6. The structure of the Tyr6, Tyr8, Tyr13, and Tyr15 region in proty4. Tyr6 interacts attractively with Tyr13 and Tyr15, with average energies equal to -19 and $-22 \mathrm{~kJ} \mathrm{~mol}^{-1}$, respectively. Tyr13 interacts with Tyr6 and Tyr8, both showing average energies equal to $-19 \mathrm{~kJ} \mathrm{~mol}^{-1}$.

The average $\mathrm{CM}$ distances between the aromatic ring of these tyrosine residues, as well as their interplanar angles and interaction energies, are listed in Table 6. Figure 7A depicts the aromatic ring CM distance histograms for Tyr6-Tyr15 and Tyr8-Tyr13 residues where the presence of just one peak indicates only one preferential conformation. The histograms for Tyr6-Tyr8, Tyr6-Tyr13 and Tyr13-Tyr15 residues (Figure 7B) show two peaks indicating that the Tyr6-Tyr15 pair, whose CM distance remains close to one average value, oscillates in a back and forth movement close to the Tyr8-Tyr13 pair, as seen in Figure 7C and D. The peculiar packing of such intercalated rings favors significant attractive interaction energy between them. In combination with the HBs (Table 1), the average CM distances constitute the interactions responsible for the maintenance of the $\beta$-hairpin structure of proty 4 . 
Table 6. Proty4. Average center of mass distances, interplanar angles and interaction energies between specific tyrosine pairs.

\begin{tabular}{lccc}
\hline Residues & $\begin{array}{c}\text { Average center of mass distance (nm) } \\
\text { Angles } \\
(\text { degree })\end{array}$ & $\begin{array}{c}\text { Average energy } \\
\left(\mathrm{kJ} \mathrm{mol}^{-1}\right)\end{array}$ \\
\hline Tyr6-Tyr15 & $0.47 \pm 0.08$ & $38 \pm 20$ & $-22 \pm 5$ \\
Tyr6-Tyr13 & $0.71 \pm 0.07$ & $25 \pm 13$ & $-14 \pm 3$ \\
Tyr6-Tyr13 & $0.48 \pm 0.07$ & $95 \pm 18$ & $-24 \pm 4$ \\
Tyr8-Tyr13 & $0.53 \pm 0.08$ & $54 \pm 19$ & $-19 \pm 4$ \\
\hline
\end{tabular}

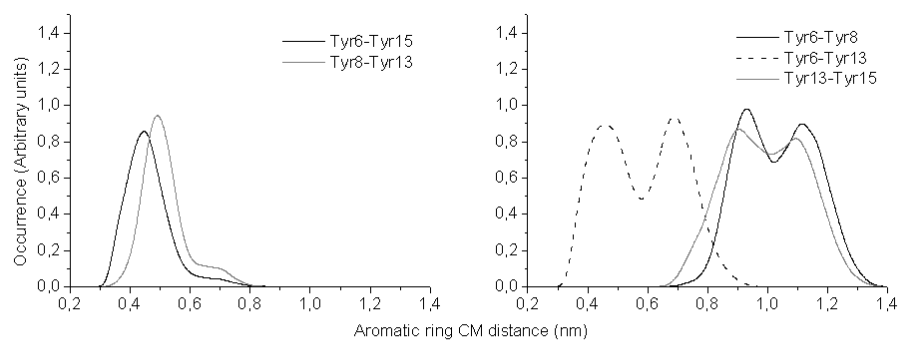

(a)

(b)

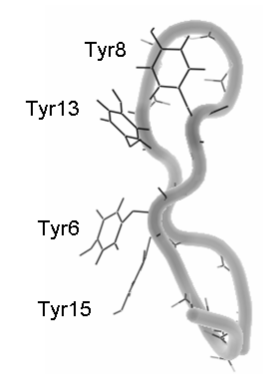

(c)

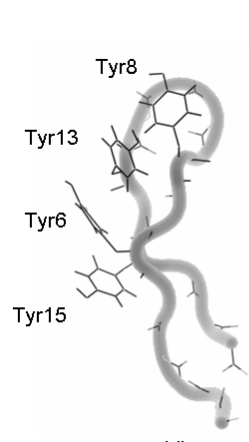

(d)

Figure 7. Proty4. (a) Aromatic ring center of mass (CM) distance histograms for Tyr6-Tyr15 and Tyr8-Tyr13 residues. (b) Aromatic ring center of mass distance histograms for Tyr6-Tyr8, Tyr13-Tyr15 and Tyr6-Tyr13 residues indicating two preferential positions. (c) and (d) The simultaneous back and forth movement of Tyr6 and Tyr15 residues close to the Tyr8/Tyr13 pair.

The small average distances between the $\mathrm{C}_{\alpha}$ atoms of the cysteine residues are due to covalent disulfide bridges linking antiparallel $\beta$-strands (Srinivasan et al., 1990). For gomesin, the average distances between the $\mathrm{C}_{\alpha}$ belonging to both disulfide bridges, namely Cys2-Cys15 and Cys6-Cys11, as shown in Figure 8A, are $0.37 \pm 0.04$ and $0.36 \pm 0.02 \mathrm{~nm}$, respectively, which is in good agreement with the experimental values, namely $0.375 \pm 0.010 \mathrm{~nm}$ for both bridges (Mandard et al., 2002). As for gomy 4 , average $\mathrm{C}_{\alpha}$ distances of $0.5 \pm 0.2 \mathrm{~nm}$ were found for both corresponding Tyr-Tyr (Figure 8B). Therefore, the corresponding original inter-residue contacts are preserved, but the variants exhibit increased flexibility, as suggested by the standard deviation values. 


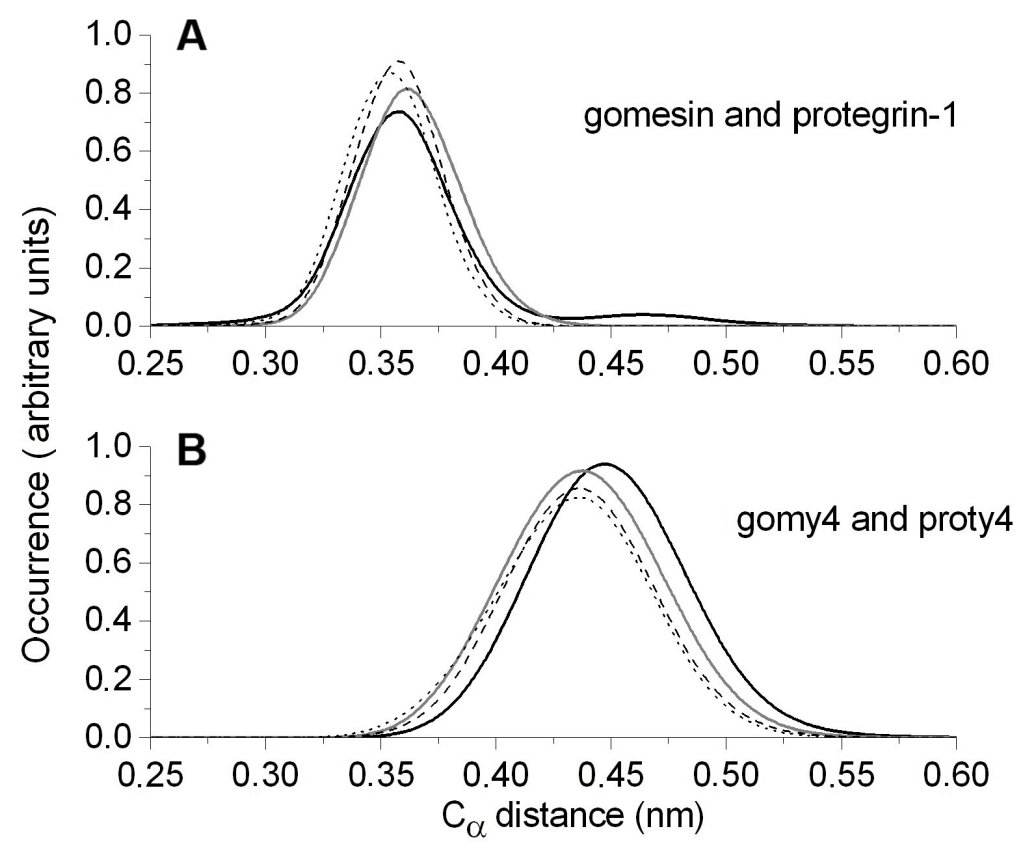

Figure 8. Distributions of the distances between pairs of $\mathrm{C}_{\alpha}$ for A. gomesin: Cys2-Cys15 (dashed line) and Cys6Cys11 (dotted line), and for protegrin-1: Cys6-Cys15 (gray line) and Cys8-Cys13 (solid line); B. gomy4: Tyr2-Tyr15 (dashed line) and Tyr6-Tyr11 (dotted line), and for proty4: Tyr6-Tyr15 (gray line) and Tyr8-Tyr13 (solid line).

The equivalent MD calculation gives values for protegrin- 1 of $0.36 \pm 0.02 \mathrm{~nm}$ for the Cys6-Cys 15 bridge, and $0.35 \pm 0.03 \mathrm{~nm}$ for the Cys8-Cys13 bridge. Both values are also in good agreement with experimental values (Fahrner et al., 1996), namely $0.35 \pm 0.01$ and 0.38 \pm 0.03 , respectively. As for the variant proty4, the corresponding $\mathrm{C}_{\alpha}$ distances are $0.44 \pm 0.03$ $\mathrm{nm}$ for Tyr6-Tyr15, and $0.43 \pm 0.03 \mathrm{~nm}$ for Tyr8-Tyr13, also indicating that the original interresidue contacts are preserved.

Comparing the distribution of the average distance between pairs of $\mathrm{C}_{\alpha}$ for gomesin and protegrin- 1 with the corresponding variants, shown in Figure $8 \mathrm{~A}$ and $\mathrm{B}$, we see that there is a relatively small displacement of the distribution peak toward higher values, but in the case of both variants, there is a broadening of the distributions.

In the particular case of gomy4, the dispersion of the distances is comparatively very large, although their values remain smaller than $0.6 \mathrm{~nm}$ for about $75 \%$ of the simulation time. The reason for such behavior may be the fact that the interactions between gomy4 tyrosine residues occur more separately along the chain compared with proty4. Indeed, while in protegrin-1 there is only one residue between Cys6/Cys8 and Cys13/Cys15, in gomesin there are three residues separating Cys2/Cys6 and Cys11/Cys15. These differences do not exert any influence in the case of the natural peptides because covalent disulfide bonds are short and very strong. But for the mutants, in which cysteines have been substituted for tyrosines, with much lower interaction energy, details on the spatial arrangement are determinant. Indeed, in proty4 the four tyrosine residues arrange themselves by intercalating Tyr15, Tyr6, Tyr13, and 
Tyr8 in a "sandwich-like" pattern (Figure 6), enhancing the effectiveness of their attractive interaction. The net result is that the attractive energy is very effective, producing a slightly more rigid structure than that of gomy4. In this latter case, Tyr15 interacts with PCA1 and Tyr2 simultaneously, and the expected interaction between the Tyr6 and Tyr11 residues is absent. The energetic scenario only gets better because Tyr7 and Tyr14 become strategically close, producing a new Tyr-Tyr attractive interaction.

For the gomesin Cys2-Cys15 linkage, the distance distribution shows a smooth peak at about $0.47 \mathrm{~nm}$, as shown in Figure 8A. This means that during a short period of time (of about $0.4 \mathrm{~ns}$ ) these two residues are separated by a larger distance. The change in the Cys-Cys $\mathrm{C}_{\alpha}$ distance goes along with changes in the proper dihedral angle, $\alpha$, defined by the atoms $\mathrm{CB}(\mathrm{Cys} 2), \mathrm{SG}(\mathrm{Cys} 2), \mathrm{SG}(\mathrm{Cys} 2)$ and $\mathrm{CB}(\mathrm{Cys} 15)$, which varies from $170^{\circ}$ to $90^{\circ}$ approximately, as seen in the histogram of Figure 9 (black bars). The corresponding potential, calculated with the Gromos 96 force field (Van Gunsteren et al., 1996) parameters (the solid line in Figure 9), shows two maxima: at $0^{\circ}$ and $180^{\circ}$, and one minimum at $90^{\circ}$. The MD values for $\alpha$ are more frequently included in the $150^{\circ}$ to $180^{\circ}$ range exactly in the maximum potential energy region, but the values that are also observed in the $90^{\circ}$ region coincide with the minimum of the $\alpha$ potential energy. These results indicate that two opposite effects are competing: the first is the natural tendency for $\alpha$ to assume values that reduce the corresponding potential energy, while the second effect is due to the inadequacy of the disulfide bridge to reduce the tensions in the $\mathrm{CB}(\mathrm{Cys} 2), \mathrm{SG}(\mathrm{Cys} 2), \mathrm{SG}(\mathrm{Cys} 2), \mathrm{CB}(\mathrm{Cys} 15)$ and neighboring region (Santiveri et al., 2008). At this point, the two main consequences are the hindering of the natural tendency to decrease the torsion angle energy and the hindering of HB formation.

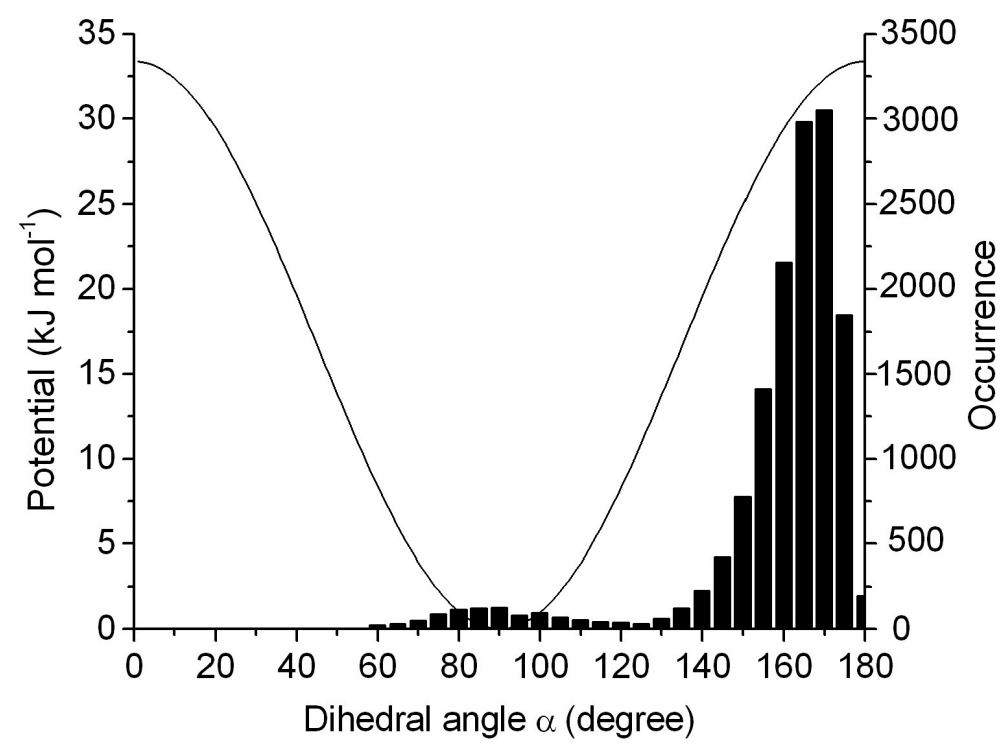

Figure 9. The energy (solid line) and occurrence (histogram) for the proper dihedral angle, $\alpha$, determined by the atoms $\mathrm{CB}(\mathrm{Cys} 2), \mathrm{SG}(\mathrm{Cys} 2), \mathrm{SG}(\mathrm{Cys} 2)$, and $\mathrm{CB}(\mathrm{Cys} 15)$. 


\section{The average structures}

The experimental structures found in the PDB for gomesin and protegrin-1 can be used to determine a kind of average structure for each corresponding peptide. Figure 10 shows the RMSD between all possible pairs of solutions taken from the set of twenty structures for protegrin-1. Most of the structures are in good agreement: about $67 \%$ of the distinct structural pairs have an RMSD $\leq 1.0 \mathrm{~nm}$, and about $95 \%$ have an RMSD $\leq 1.6 \mathrm{~nm}$. The situation for gomesin is equivalent. Therefore, the idea of "average structure" makes sense.

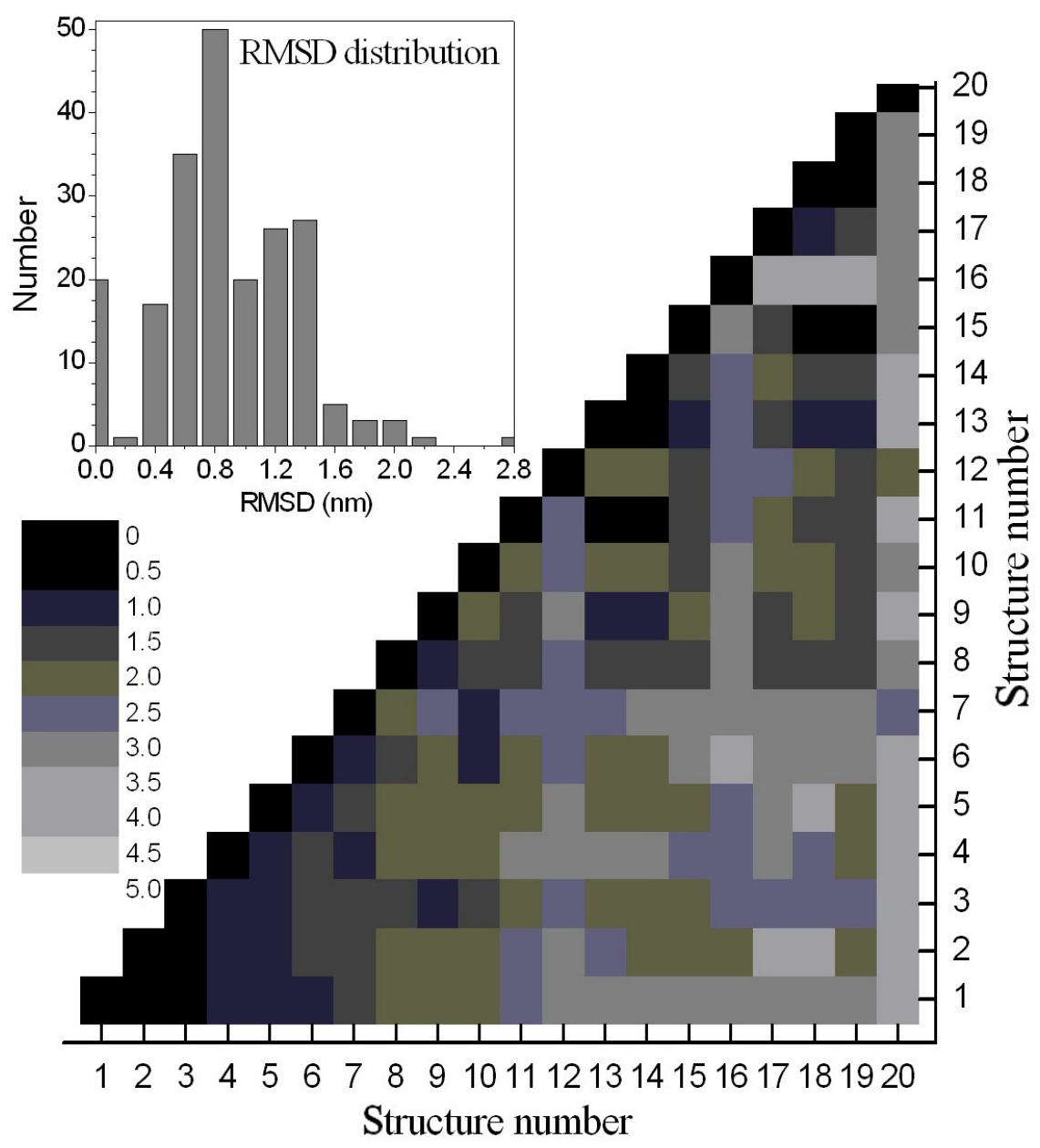

Figure 10. Protegrin-1. Root mean square deviation (RMSD, $\AA$ ) map for all 190 pairs of solution, from the set of 20 solutions from the PDB; different shades of gray identify distinct RMSD intervals. The diagonal, black squares refer to structural self-comparisons $(\mathrm{RMSD}=0$ ). Lower (full) row gives the RMSD for fittings of the first structure, as appears in the PDB, with all the other structures, which were re-ordered according to decreasing RMSD values. The inset shows the histogram for RMSD distribution: about $67 \%$ of distinct pairs show an RMSD $<1.0 \mathrm{~ns}$; the first column corresponds to the 20 structural self-comparisons. 
Figures 11 and 12 depict the superposition of the average structures of the natural peptides and their respective mutants from two different perspectives. Figure 11A and B show that the absence of disulfide bridges causes only a small torsion in the gomy4 and proty4 turning region.

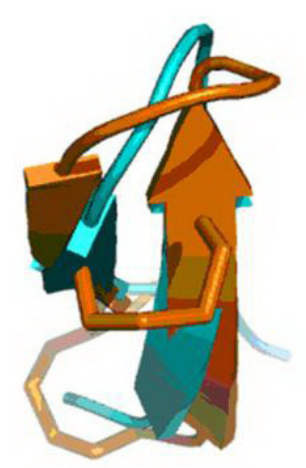

A

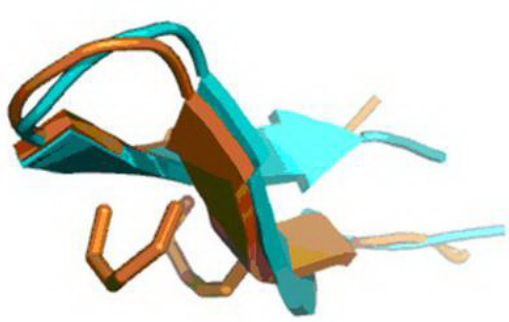

B

Figure 11. The turning regions in gomesin and protegrin-1 and their mutants: Superposition of the average structures: A. gomesin (orange) and gomy4 (blue) structures, RMSD $=0.27 \mathrm{~nm}$; B. protegrin-1 (orange) and proty4 (blue) structures, RMSD $=0.19 \mathrm{~nm}$.

Figure 12 depicts the corresponding side views of the average structures of gomesin and gomy4. Figure 12A shows the great structural similarity between the sequences of residues from PCA1 up to Lys8, while Figure 12B focuses on the sequence from Val12 to Arg18, emphasizing the region that shows the largest deviations.
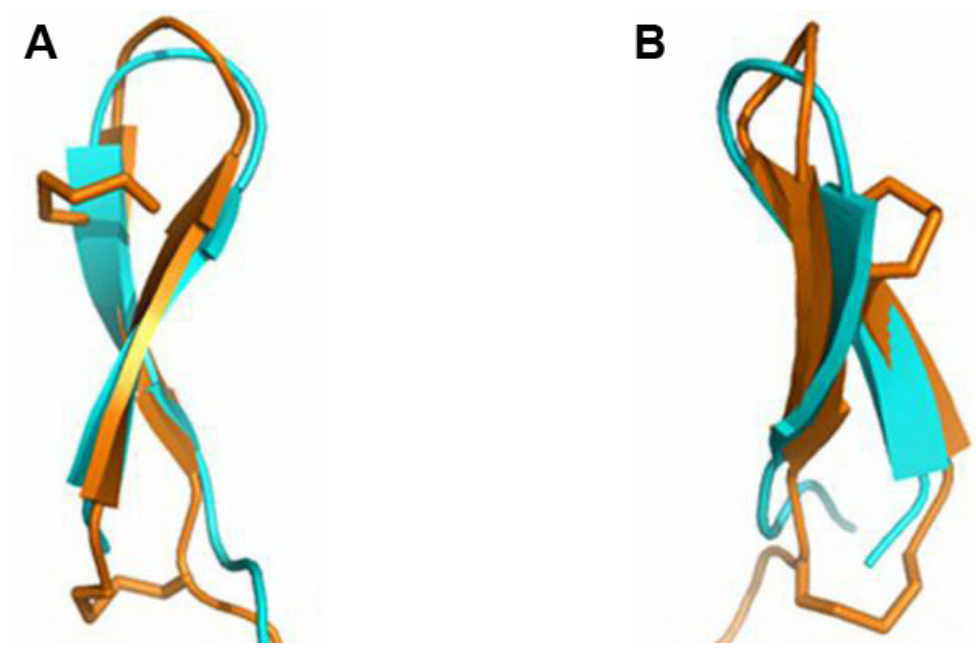

Figure 12. Gomesin and gomy4: side views. Superposition of gomesin (orange) and gomy4 (blue) average structures emphasizing the lateral region of the two peptides: A. residues from PCA1 up to Lys8 are shown in the first plane $(\mathrm{RMSD}=0.082 \mathrm{~nm}$ for this segment); B. residues from Cys11 (Tyr11 in gomy4) up to Arg 18 are shown in the first plane (RMSD $=0.107 \mathrm{~nm}$ for this segment). 
Similarly, Figure 13 presents the lateral view of the superposition of protegrin-1 and proty4 average structures, also showing striking overall similarity.
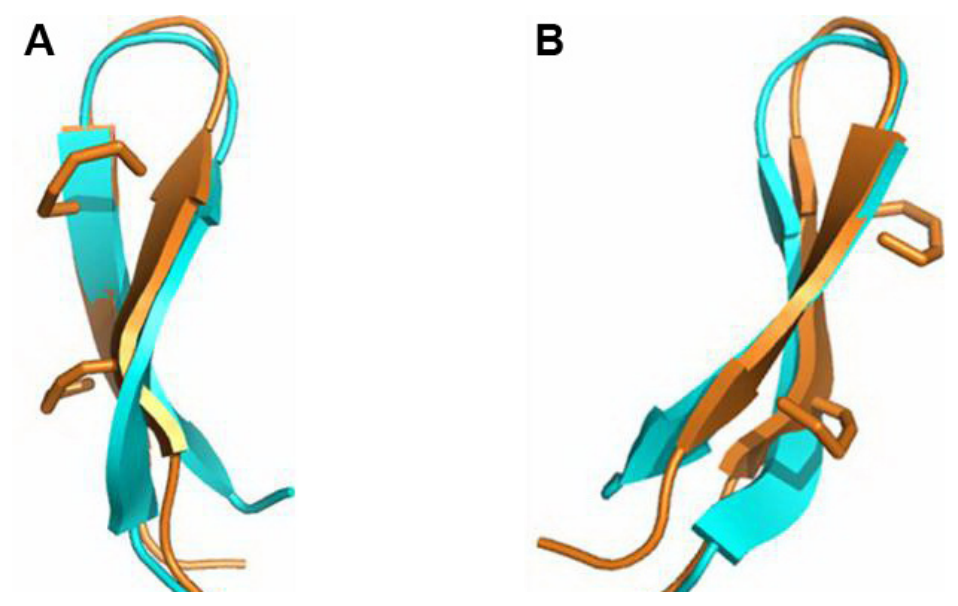

Figure 13. Protegrin-1 and prot4: side views. Superposition of protegrin-1 (orange) and proty4 (blue) average structures: A. residues from Arg1 up to Tyr7 are in the first plane (RMSD $=0.102 \mathrm{~nm}$ for this segment); B. residues from $\operatorname{Arg} 11$ up to $\operatorname{Arg} 18$ are in the first plane (RMSD $=0.124 \mathrm{~nm}$ for this segment).

\section{DISCUSSION}

The agreement between experimental and theoretical (MD simulations) structural and HB data ensures that the parameters and force field used in our study describe the systems under consideration quite well. For both peptides, gomesin and protegrin-1, the residues with two positive or two negative Ramachandran $(\Phi, \Psi)$ angles, located at the turn region of the $\beta$-strand, as predicted by our MD simulations, display more than one preferential angle pair (multimodal distribution). Such structural flexibility - despite the disulfide bridges and crossstrand HBs - permits systematic and specific conformational exchanges, which may help explain their broad-spectrum antimicrobial activity.

The simulations of the variants proty4 and gomy4 demonstrate the importance of the cross-strand HBs for the maintenance of the structural stability in aqueous solution, since most of them were preserved and strengthened (higher occurrence). The residues Cys and Tyr are sterically very different, but their hydrophobic levels are comparable: both are hydrophobic (free energy cost to move a residue from a non-polar environment into water), exhibiting amphipathy values of 3.8 and 21.3, respectively, as reported by Mazzé et al. (2005). Besides the hydrophobic effect, both residues interact attractively with the solvent through-short range interactions, but they are stronger for Tyr than for Cys because in the former case such interactions are roughly proportional to the number of atoms in the residue (Mazzé et al., 2005), and the hydroxyl polar group in Tyr increases such attractive interactions. In addition, the aromatic rings of the Tyr residues interact attractively, keeping the residues close to each other. However, the neat result is an effective stability of the structure around the Tyr residues. This fact may explain the resulting untidy stability of the variants and, because of the extra hydrophobic pack- 
ing factor introduced by Tyr residues, it also suggests that the variants gomy4 and proty4 may accomplish a completely successful folding process, since the covalent disulfide bonds are not necessarily required for correct folding in many cases (Eisenberg and McLachlan, 1986).

Both gomesin and protegrin-1 show the unusual combination of positive $\Phi$ and $\Psi$ angles in the Lys8 and Arg10 residues, respectively, which are located in the turn region (the head) of their respective $\beta$-hairpin motifs. In the turn region, the angles in the respective variants undergo a qualitative change, assuming a combination of positive and negative values for $\Phi$ and $\Psi$, respectively, but still exhibiting a high degree of similarity between the respective natural and variant species. Therefore, because the residues Lys8 and Arg10 are active points in the molecule, such subtle behavior may promote an increase, or decrease, in peptide activity, affecting their antimicrobial properties and/or their hemolytic effect. This deserves a specific experimental investigation.

\section{CONCLUSION}

Disulfide bridges are considered to be important in the maintenance of the conformation of many antimicrobial peptides, with evident consequences on their bioactivity. The influence of disulfide bridges on the structures of the gomesin and protegrin-1 antimicrobial peptides was investigated by exchanging the two disulfide bridges by tyrosine interactions with residues placed at the same positions of the cysteine residues. Our results show that no major changes in the structures of the peptides are observed, but the variants have greater flexibility than their respective natural peptides. The consequences of these alterations on the antimicrobial activity of the gomesin and protegrin-1 antimicrobial peptides must be experimentally investigated. Another question is the generality of our present results: Can they be extended to other antimicrobial peptides containing disulfide bridges?

\section{ACKNOWLEDGMENTS}

The authors are thankful to Coordenação de Aperfeiçoamento de Pessoal de Nível Superior (CAPES), Conselho Nacional de Desenvolvimento Científico e Tecnológico (CNPq) and Fundação de Amparo à Pesquisa do Estado de São Paulo (FAPESP) for financial support.

\section{REFERENCES}

Berendsen HJC, Postma JPM, van Gunsteren WF, Dinola A, et al. (1984). Molecular-Dynamics with coupling to an external bath. J. Chem. Phys. 81: 3684-3690.

Berendsen HJC, Grigera JR and Straatsma TP (1987). The missing term in effective pair potentials. J. Phys. Chem. 91: 6269-6271.

Chan DI, Prenner EJ and Vogel HJ (2006). Tryptophan- and arginine-rich antimicrobial peptides: structures and mechanisms of action. Biochim. Biophys. Acta 1758: 1184-1202.

Che Q, Zhou Y, Yang H, Li J, et al. (2008). A novel antimicrobial peptide from amphibian skin secretions of Odorrana grahami. Peptides 29: 529-535.

Daffre S, Miranda A, Miranda MTM, Bulet P, et al. (2001). Peptídeos antibióticos produzidos por aracnídeos. Biotecnol. Cienc. Desenvolv. 23: 48-55.

Darden T, York D and Pedersen L (1993). Particle Mesh Ewald - an N.Log(n) method for Ewal sums in large systems. $J$. Chem. Phys. 98: 10089-10092.

Eisenberg D and McLachlan AD (1986). Solvation energy in protein folding and binding. Nature 319: 199-203.

Fahrner RL, Dieckmann T, Harwig SS, Lehrer RI, et al. (1996). Solution structure of protegrin-1, a broad-spectrum antimicrobial peptide from porcine leukocytes. Chem. Biol. 3: 543-550. 
Fazio MA, Oliveira VX Jr, Bulet P, Miranda MT, et al. (2006). Structure-activity relationship studies of gomesin: importance of the disulfide bridges for conformation, bioactivities, and serum stability. Biopolymers 84: 205-218.

Guex N and Peitsch MC (1997). SWISS-MODEL and the Swiss-PdbViewer: an environment for comparative protein modeling. Electrophoresis 18: 2714-2723.

Hess B, Bekker H, Berendsen HJC and Fraaije JGEM (1997). LINCS: a linear constraint solver for molecular simulations. J. Comput. Chem. 18: 1463-1472.

Lam KL, Ishitsuka Y, Cheng Y, Chien K, et al. (2006). Mechanism of supported membrane disruption by antimicrobial peptide protegrin-1. J. Phys. Chem. B 110: 21282-21286.

Mandard N, Bulet P, Caille A, Daffre S, et al. (2002). The solution structure of gomesin, an antimicrobial cysteine-rich peptide from the spider. Eur. J. Biochem. 269: 1190-1198.

Mazzé FM, Fuzo CA and Degrève L (2005). A new amphipathy scale I. Determination of the scale from molecular dynamics data. Biochim. Biophys. Acta 1747: 35-46.

Miyamoto S and Kollman PA (1992). Settle - an analytical version of the Shake and Rattle algorithm for rigid water models. J. Comput. Chem. 13: 952-962.

Prates MV and Bloch Júnior C (2000). Peptídeos antimicrobianos: uma alternativa no combate a microorganismos resistentes. Biotecnol. Cienc. Desenvolv. 17: 30-36.

Ramamoorthy A, Thennarasu S, Tan A, Gottipati K, et al. (2006). Deletion of all cysteines in tachyplesin I abolishes hemolytic activity and retains antimicrobial activity and lipopolysaccharide selective binding. Biochemistry 45: 6529-6540.

Rao AG (1999). Conformation and antimicrobial activity of linear derivatives of tachyplesin lacking disulfide bonds. Arch. Biochem. Biophys. 361: 127-134.

Santiveri CM, Leon E, Rico M and Jimenez MA (2008). Context-dependence of the contribution of disulfide bonds to beta-hairpin stability. Chemistry 14: 488-499.

Silva PI Jr, Daffre S and Bulet P (2000). Isolation and characterization of gomesin, an 18-residue cysteine-rich defense peptide from the spider Acanthoscurria gomesiana hemocytes with sequence similarities to horseshoe crab antimicrobial peptides of the tachyplesin family. J. Biol. Chem. 275: 33464-33470.

Srinivasan N, Sowdhamini R, Ramakrishnan C and Balaram P (1990). Conformations of disulfide bridges in proteins. Int. J. Pept. Protein Res. 36: 147-155.

Van Der Spoel D, Lindahl E, Hess B, Van Buuren AR, et al. (2004). Gromacs User Manual version 3.2. [http://www. gromacs.org]. Accessed September 29, 2005.

Van Der Spoel D, Lindahl E, Hess B, Groenhof G, et al. (2005). GROMACS: fast, flexible, and free. J. Comput. Chem. 26: 1701-1718.

Van Gunsteren WF, Billeter SR, Eising AA, Hünenberger PH, et al. (1996). Biomolecular Simulation: The GROMOS96 Manual and User Guide, VdF: Hochschulverlag AG an der ETH Zürich and BIOMOS b.v. Groningen, Zürich, ISBN 3728124222.

Zasloff M (2002). Antimicrobial peptides of multicellular organisms. Nature 415: 389-395. 